ENCYCLOPÉDIE Encyclopédie berbère

BERBERE

$35 \mid 2013$

35| Oasitae - Ortaïas

\title{
Orientation (Linguistique / Syntaxe)
}

Salem Chaker

\section{OpenEdition}

Journals

Édition électronique

URL : https://journals.openedition.org/encyclopedieberbere/2828

DOI : 10.4000/encyclopedieberbere.2828

ISSN : 2262-7197

\section{Éditeur}

Peeters Publishers

\section{Édition imprimée}

Date de publication : 1 juin 2013

Pagination : 5807-5819

ISBN : 978-2-7584-0184-1

ISSN : 1015-7344

\section{Référence électronique}

Salem Chaker, " Orientation (Linguistique / Syntaxe) », Encyclopédie berbère [En ligne], 35 | 2013,

document 025, mis en ligne le 12 mars 2021, consulté le 17 février 2022. URL : http://

journals.openedition.org/encyclopedieberbere/2828; DOI : https://doi.org/10.4000/

encyclopedieberbere.2828

Ce document a été généré automatiquement le 17 février 2022.

(c) Tous droits réservés 


\title{
Orientation (Linguistique / Syntaxe)
}

\author{
Salem Chaker
}

1 En linguistique berbère, la notion d'orientation fait référence à deux ordres de phénomènes totalement distincts :

- Les morphèmes $-d(d)$ et $-n$, affixes du verbe, dénommées "modalités d'orientation" par Bentolila (1969) ou, plus traditionnellement, "particules de mouvement" ou "de rection" (Basset 1952/1969, p. 36).

-L'organisation syntaxique de l'énoncé verbal, i.e. les relations de son prédicat avec les deux principaux participants ou actants du procès, l'agent et le patient.

\section{Les particules d'orientation $-d(d)$ et $-n$}

2 Il s'agit d'un micro-paradigme grammatical de morphèmes affixes du verbe qui apportent à celui-ci une information sémantique relative à l'orientation du procès par rapport au locuteur et à l'auditeur. Les descriptions anciennes (Basset-Picard 1948, p. 135-146; Aspinion 1953, p. 217-211...) mettaient surtout en valeur leur valeurs de déplacement dans l'espace :

-d étant défini comme "particule d'approche/rapprochement", marquant un mouvement vers le locuteur ou son lieu (réel ou supposé),

$-n$, défini comme "particule d'éloignement", indiquant un mouvement d'éloignement, généralement vers l'auditeur ou son lieu (réel ou supposé).

Cette description classique est globalement suffisante pour les verbes référant intrinsèquement à un déplacement ou un mouvement ; ainsi avec le verbe ddu, "aller" :

\begin{tabular}{|l|l|}
\hline$y \partial d d a$ & « il est parti/allé » (voire, dans certains parlers, « il est perdu ») \\
\hline$y \partial d d a-d$ & il est allé vers ICI = « il est venu » \\
\hline$y \partial d d a-n$ & il est allé vers LA-BAS = « il y est allé, il est allé chez toi... » \\
\hline
\end{tabular}


Mais A. Basset était déjà très conscient de la complexité des choses qui écrivait :

«Mais le jeu de ces particules, l'un des plus délicats de la langue, est infiniment plus complexe et nous restons souvent, pour la particule $d$, en présence d'emplois dont nous ne saisissons pas bien la raison d'être. » (1952/1969, p. 36).

5 Car la difficulté - et la spécificité du berbère - réside dans le fait que ces particules peuvent se combiner avec quasiment n'importe quel verbe, qu'il renvoie à un mouvement/déplacement ou non ; ainsi :

\begin{tabular}{|c|c|}
\hline Уәс̌с̌A & $=$ = il a mangé $»$ \\
\hline \multirow[t]{2}{*}{ уәс̌⿻̆丶 } & il a mangé VERS ICI \\
\hline & $=$ «il a/avait (déjà) mangé (ailleurs) avant de venir (ici)» \\
\hline \multirow[t]{2}{*}{ уәс̌с̌A-П } & il a mangé VERS LA-BAS \\
\hline & $=$ « il a/avait (déjà) mangé avant d'y aller/avant d'aller chez toi... » \\
\hline
\end{tabular}

6 Les travaux plus récents (Bentolila 1969 ; Chaker 1983, § 15.30-15.33 ; Aït-Ahmed 1992...) ont mis en évidence, à partir de nombreux exemples n'impliquant aucun déplacement ou mouvement physique, que ces morphèmes ont une valeur quasiment "modale*" et concernent en réalité l'implication des protagonistes de l'acte de parole, locuteur et auditeur, dans le procès verbal. Les énoncés :

\begin{tabular}{|l|l|l|}
\hline (1a) ilul wayyur & elle-est née la lune & $=$ « la (nouvelle) lune est apparue » \\
\hline (1b) ilul wəqšiš & il-est né garçon & $=$ « un garçon est né » \\
\hline (1c) yurəw uzəmmur & il-a produit olivier(s) & $=$ « les oliviers ont produit (beaucoup) » \\
\hline
\end{tabular}

7 s'opposeront nettement à

\begin{tabular}{|l|l|l|}
\hline $\begin{array}{l}\text { (2a) ilul-dd } \\
\text { wayyur }\end{array}$ & la lune est née ICI & $=$ «la (nouvelle) lune (nous) est apparue » \\
\hline (2b) ilul-dd wəqšišs & $\begin{array}{l}\text { un garçon est né } \\
\text { ICI }\end{array}$ & $=$ « un garçon (nous) est né » \\
\hline $\begin{array}{l}\text { (2c) yurəw-dd } \\
\text { uzəmmur }\end{array}$ & $\begin{array}{l}\text { il-a produit-ICI } \\
\text { olivier(s) }\end{array}$ & $\begin{array}{l}\text { a "les oliviers (nous) ont (beaucoup) produit » = «nous } \\
\text { avons eune belle récolte d'olives » }\end{array}$ \\
\hline
\end{tabular}

8 En (1), l'énoncé est "neutre" et exprime un simple constat, parfaitement distancié, du locuteur sur un événement; en (2), le locuteur est clairement impliqué, il se pose comme bénéficiaire $d u$ procès: il s'agit d'un événement qui le concerne personnellement. 
9 On voit que la notion d'orientation dépasse largement celle de déplacementmouvement et touche au fond à la relation Locuteur-Auditeur/Procès, indépendamment de toute référence spatiale. Pour englober l'ensemble des configurations possibles, on proposera les formulations de "Orientation vers le locuteur"/“Orientation vers l'auditeur”.

10 Ces deux morphèmes sont attestés dans la généralité du berbère, mais il a été noté depuis longtemps (notamment Basset \& Picard 1948; Basset 1952/1969) que -d est beaucoup plus fréquent que $-n$ et que de nombreux parlers ont même abandonné l'usage de $-n$, ou n'en gardent que des traces résiduelles, y compris au sein de dialectes comme le kabyle qui, pris globalement, sont censés connaître les deux particules. Cette tendance lourde n'est guère surprenante si l'on retient la valeur "modale" définie cidessus : dans l'acte d'énonciation, l'orientation vers le locuteur a naturellement toutes les chances d'être beaucoup plus fréquente et plus affirmée que l'orientation vers l'auditeur : l'acte de parole est d'abord un acte subjectif !

11 Au plan morphologique, la forme de base du morphème $-d$, semble bien être tendue [dd], comme l'indiquent sa réalisation dans plusieurs dialectes (notamment en chleuh) et le fait qu'elle demeure toujours occlusive dans les parlers connaissant la spirantisation* (kabyle, rifain...). Ce sont sans doute son apparition habituelle en position finale du syntagme verbal et sa haute fréquence qui expliquent la tendance à l'affaiblissement articulatoire et sa réalisation [d] (occlusive simple) dans des dialectes comme le kabyle. Certains parlers (chleuh encore) connaissent aussi une variante tendue $-n n$.

12 Morphèmes constitués d'un seul phonème, toujours intégrés dans le syntagme verbal, ces particules entrent très régulièrement en contact direct avec d'autres éléments consonantiques appartenant à d'autres morphèmes : on aura donc très souvent, et dans tous les dialectes, des variantes post-consonantiques vocalisées en /i/ (-id / -in), pour éviter les successions de consonnes difficilement prononçables sans élément vocalique :

yəfka-d idrimən, il a donné-ICI argent = « il (nous) a donné de l'argent » 
"Attracteur" + (Pron. Pers. Ind.) + (Pron. Pers. Direct) + (Particule d'orientation) +

VERBE

ur as- $t$ - id- yəfki = Nég. à lui- le- ICI- il a donné = « il ne le lui a pas donné »

18 Enfin, toujours au plan morphologique, on notera que ces particules d'orientation du procès ont un lien évident avec certains morphèmes déictiques suffixes du Nom ("démonstratifs") :

NOM $-(a) d=$ marquant la proximité par rapport au locuteur = “-ci”, "celui-ci”

argaz-ad = homme-ci = "cet homme" (chleuh)

NOM -(i)n= marquant l'éloignement par rapport au locuteur =“-là", 'celui-là/là bas“

argaz-in "homme-là) = "l'homme-là bas"

19 Le couple de déictiques post-nominaux est opportunément illustré par une belle formule de voeu, archaïsante, dite à un Kabyle candidat à l'émigration :

A k-yəldi Ŗəbbi tiwwura $g^{w}$ əmmad-in, $g^{w}$ ommad-ad! = "Que Dieu t'ouvre les portes sur l'autre rive ( $g^{w}$ əmmad-in) comme sur cette rive ( $g^{w}$ əmmad-ad) !"

20 En diachronie, ces morphèmes $-d$ et $-n$ devaient donc être polyvalents et déterminer indifféremment le Nom et le Verbe et appartenir à un système unique de déictiques (cf. Galand 2010, p. 91).

21 2. Syntaxe de l'énoncé verbal berbère : la non orientation du prédicat, une tendance lourde.

22 Dans le champ des études berbères, S. Chaker a été le premier (dès sa thèse de doctorat de 1973) à avoir essayé d'apporter une explication syntaxique objective à un phénomène, connu bien antérieurement et précisément pointé par André Basset dans sa belle synthèse de 1952 :

« ... pourquoi l'expression du passif par une forme dérivée quand, en base, [...] la forme simple par elle-même, a généralement les trois valeurs d'actif, passif et réfléchi? Et si parfois certains verbes se refusent aux trois valeurs, c'est la valeur passive [...] qui est exclusive de la forme simple, l'actif étant alors exprimé par la forme à sifflante » $(1952$, p. 13). 
grand nombre de verbes simples du point de vue de leur orientation par rapport aux participants du procès. C'est ce qu'on appelait traditionnellement la "valeur passive du verbe simple" qui existe pour de très nombreux lexèmes verbaux usuels. Des verbes comme kkəs "enlever/ôter", gzəm "couper", qqən "attacher/lier", krəz, "labourer"... peuvent avoir le sens de "être enlevé", "être coupé", "être attaché", "être labouré", cela sans aucune modification formelle, i.e. sans passivation.

Là encore, le phénomène n'est pas du tout inconnu ailleurs puisqu'il renvoie à la catégorie des "verbes mixtes", "réversibles" ou "symétriques" d'une langue comme le français (cf. notamment Dubois 1967, p. 25, 87, 110 et sq., 121-122) : "casser" $\rightarrow$ "il casse la branche"/"la branche casse"; couler $\rightarrow$ "il coule le bateau"/"le bateau coule"; on en trouve également de nombreux exemples en anglais... La spécificité berbère réside, là encore, dans l'ampleur du phénomène: plusieurs centaines de verbes simples, appartenant au vocabulaire fondamental, partagent ce trait (Chaker 1983, p. 300-301 et 1984, p. 209-210): situation différente donc d'une langue comme le français où le phénomène paraît marginal. Et la plupart d'entre eux peuvent être considérés comme logiquement processifs et supposant un agent et un patient distincts - et souvent un instrument - pour qu'il y ait réalisation du procès : il suffit de parcourir un dictionnaire bilingue berbère-français, pour se convaincre de l'ampleur du phénomène et surtout de ce qu'il n'est pas du tout lié à une zone sémantique particulière ; en touareg, en kabyle, en tamazight..., une kyrielle de verbes ont pour équivalents français à la fois un verbe transitif et son correspondant dit "passif » (“construire/être construit”...). Un énoncé comme :

gəzmen ibərdan : couper (+ prétérit)-ils chemins/routes est parfaitement ambigu et pourra signifier indifféremment: "les routes sont coupées » ou « ils ont coupé les routes ». Sans complément supplémentaire, le locuteur retiendra d'ailleurs plutôt la première interprétation : « les routes sont coupées ».

C'est ce phénomène syntaxique qui permet de comprendre comment un verbe comme əg, traduit habituellement par "faire", mais qui est en réalité un verbe de type mixte (donc aussi "être fait") a pu devenir un véritable verbe "être" dans certains dialectes (chleuh notamment, mais pas uniquement); partout, le verbe connaît des emplois transitifs, avec "sujet" agent et objet patient :

Kab. : tga-d. imənsi : elle-a fait ICI dîner = « elle a fait/préparé le dîner » et des emplois strictement intransitifs, avec un seul participant, patient/attributaire :

Kab. : amək tga təmyart ? : comment elle-est (faite) vieille ? = « comment va la vieille (femme)?»

Certaines variétés régionales de la langue ont généralisé cet usage, faisant de $ə g$ un véritable verbe copule "être", qui leur permet d'avoir des énoncés du type :

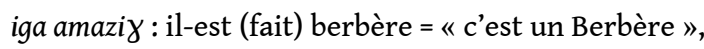

mais les bases syntaxiques de cette refonctionnalisation sont présentes dans tous les dialectes.

Un indice du caractère fondamental de ce trait pour la syntaxe du verbe berbère est le traitement des emprunts arabes et français : même les lexèmes verbaux provenant de ces langues connaissent ce fonctionnement mixte (bien sûr exclu dans la langue d'origine) : xdəm "faire/ê. fait", hudd "détruire/ê. détruit", bnu "construire/ê. construit", ppiri “opérer/ê. opéré" (médecine)... : 
ihudd. axxam, « il a détruit la maison "/ ihudd wəxxam, « la maison est détruite ", yəbna axxam «il a construit une maison» / yəbna wəxxam «la maison est construite »

34 On soulignera que l'analyse traditionnelle en termes de "valeur passive du verbe simple » était et reste répandue chez les berbérisants ; on citera, parmi bien d'autres, J. Delheure (Grammaire de la Teggargrent - Ouargla, 1987, p. 14) :

«Une remarque est à faire avant d'aborder cette étude : nombre de verbes ouarglis à la forme simple ont un sens soit actif, soir passif, soit les deux à la fois, ex. $\partial g$, être fait et faire [...]. Pour mieux dire, ces verbes peuvent être transitifs ou intransitifs... "

Il s'agit, ici comme déjà chez A. Basset, d'une formulation méthodologiquement problématique, qui risque de conduire à analyser les structures d'une langue à partir du filtre de la traduction dans une langue de travail - ici le français, mais pendant des siècles le latin! La forme est dite " passive » parce qu'elle correspond à une séquence française passive (ou à verbe "être"). Or, on doit le souligner fortement, rien dans l'énoncé berbère ne manifeste une quelconque marque de passivation du verbe. Il y a fort longtemps (Chaker 1973, 1983, 1984, 1995) que nous avons attiré l'attention sur le fait que l'existence même de formes verbales dérivées explicitement passives (par préfixation de morphèmes $t$ tw- ou $m$ - ) :

a. interdisait de considérer la forme simple (sans morphème de passivation) comme « passive $^{2} »$;

b. imposait d'analyser les formes verbales simples dans le cadre d'un système propre à la langue qui oppose formes simples à formes dérivées passives; ce qui permet de faire immédiatement apparaître la valeur d'abord stative du verbe simple, par opposition à la valeur processive/résultative de la forme dérivée passive :

yəgzəm wəbrid, il-est coupé chemin/route = « le chemin/route est coupé(e) » yəttwagzəm wəbrid, il+passif+est coupé chemin/route = «le chemin/la route a été coupé(e) ».

Le second énoncé implique un procès, un résultat et un agent extérieur (non mentionné) ; le premier constate simplement une situation.

Au plan de la linguistique générale, ce type de fonctionnement est bien connu : il s'agit de de la non-orientation du verbe - dont l'ergativité d'une langue comme le basque n'est qu'une forme particulière - , bien décrite par Tchekhoff $(1978, \S I .12)$ qui le qualifie de "disponibilité" du prédicat :

«...la non-orientation du prédicat est mise à profit par la syntaxe: puisque ni prédicat ni premier participant ne spécifient le rôle que joue celui-ci par rapport à son prédicat, la même syntaxe pourra servir à exprimer des situations référentiellement opposées, la compréhension du message étant assurée par la situation extralinguistique ou la vraisemblance. Mais il faut pour cela, bien entendu, que l'agent ne soit pas compris dans l'énoncé ; sinon, de sa présence même, découleraient les rapports de toutes les autres unités... »

En berbère, sans que le phénomène soit généralisé, le verbe a une tendance lourde à être un prédicat d'existence entretenant avec son indice personnel (et l'explicitation lexicale de celui-ci, i.e. le complément explicatif dans la terminologie de L. Galand) une relation non-spécifiée ("agent", "patient", "attributaire"). On rappellera que l'hypothèse de l'ergativité primitive du verbe en chamito-sémitique est admise par tous les grands «classiques » (par exemple, Diakonoff 1988, p. 111). Sur ce plan, le berbère 
serait donc particulièrement conservateur en ayant conservé pour un grand nombre de ses verbes cette neutralité syntaxique originelle.

\section{Les implications de la non-orientation tendancielle du verbe berbère}

Les retombées de cette tendance forte qu'a le verbe berbère à être d'abord un prédicat d'existence, non-orienté, sont considérables au plan de la syntaxe et du fonctionnement du système verbal.

\section{La prépondérance de la dérivation transitivante en S-}

41 C'est évidemment le trait de non-orientation du verbe simple qui explique que la dérivation verbale la plus fréquente, et de très loin comme le montrent tous les travaux sur la dérivation verbale (Chaker 1973, Cadi 1987, 1990), et dans tous les dialectes, est la dérivation en $S$-, dont la fonction première est de transformer un verbe intransitif en verbe orienté (transitif). Des verbes comme $n z$, irid, $x s y . . .$, ne pouvant signifier que "être vendu", "être lavé", "être éteint"..., avec construction intransitive, on sera obligé de passer par un morphème "transitivant » pour introduire l'agent, d'où zzənz (<ssənz) "vendre", ssired "laver", səxsy, "éteindre"...

On a donc affaire à une langue très particulière où la dérivation la plus massive et la plus vivante n'est pas du tout le passif, mais au contraire, ce que l'on appelait traditionnellement le "factitif», qui est en réalité un "transitivant/agentivant" (Chaker 1973).

\section{Prédicat d'existence et aspect}

Les effets de ce trait marquant de la syntaxe du verbe ne touchent pas qu'à la construction de l'énoncé verbal : ils sont également importants au niveau du système des oppositions aspectuelles. Si le verbe simple à une forte propension à être un prédicat d'existence, la valeur des aspects s'en trouvera influencée dans le sens de la stativité, qui tendra à être l'un des pôles aspectuels fondamentaux du système. Affirmant que "quelque chose est" - et non pas "s'est produit ou a été produit", une des formes aspectuelles de base du verbe aura vraisemblablement la valeur de "statif" (= état, qualité, situation constatée).

Et, effectivement, pour un nombre élevé de verbes et dans de nombreux contextes, notamment lorsque l'énoncé ne comporte qu'un seul participant, i.e. lorsqu'il n'est pas orienté et tiré vers la processivité par la syntaxe de la phrase, la valeur générale du thème de prétérit se situera beaucoup plus du côté de la stativité, de l'état constaté, que de l'accomplissement ou l'achèvement du procès (ou toute autre valeur aspectuelle). Un énoncé réduit au syntagme verbal, ou ne comportant qu'un seul actant, aura toute les chances d'exprimer un constat, sans qu'il y ait la moindre nuance de résultat d'un accomplissement d'un procès : yəbzəg (ubərnus-is), il-est mouillé (burnous-son) = « son burnous est mouillé, trempé » n'implique pour le locuteur aucune référence à un état résultant d'un procès antérieur - sauf bien entendu à remettre à l'honneur une logique sémantique prétendument 
universelle mais qui n'est en réalité que la projection des faits propres à des langues et à une tradition d'analyse "logique" particulières. Ce seront toujours des déterminations secondaires qui introduiront des valeurs de processivité et de résultat, par exemple, la particule d'orientation (cf. supra) $-d$ : 2010, voir notamment p. 219 et suivantes et \$6.8.) ne souscrivent pas à cette analyse qui pose le caractère central de la statitivité dans le système des oppositions verbales. Tout en admettant souvent le rôle originel important de l'état, ils considèrent que l'évolution globale de la langue est allée dans le sens de la processivité au détriment de la statitivité. À l'appui de cette position, on cite souvent la tendance marquée (achevée notamment au Maroc) à la disparition de la conjugaison par suffixes des verbes dits "d'état ou de qualité ", régularisés sur le modèle de conjugaison standard. Le débat est évidemment complexe et dépend largement de la méthodologie d'analyse retenue, rarement explicitée et systématique. On soulignera qu'en la matière le simple « recours au corpus ", sous la forme de quelques exemples choisis ou même aléatoires, ne parait pas en suffisant dans la mesure où il est toujours possible de trouver, dans la diversité des corpus et dans l'obscurité de leur constitution, des exemples pouvant illustrer n'importe quelle thèse. S'agissant de définir la valeur sémantique de base des formes verbales, pour notre part, nous avons toujours privilégié (depuis 1973) une démarche "minimaliste", visant à dégager le prédicat verbal de toutes les déterminations contextuelles qui peuvent l'influencer et à n'introduire que dans un second temps une typologie des contextes : comment un berbérophone interprète-il un énoncé comme yəqqur, yəbzəg, yəldi, yəqqən, yəgzəm...? On a tendance à penser que l'approche "processiviste» est induite par le poids accordé aux données spécifiquement marocaines (chez Destaing et Galand notamment), par l'importance reconnue aux indices morphologiques et, sans doute, par une conception classique du verbe, considéré comme intrinsèquement lié à la processivité - ce qu'infirment de nombreuses données berbères, sémantiques et surtout morphogénétiques. En tout état de cause, tous ces éléments et arguments font débat, en théorie comme dans leurs concrétisations spécifiquement berbères.

49 Le système des oppositions aspectuelles du berbère rappelle donc fortement celui qui est postulé pour le prototype chamito-sémitique : comme ce dernier, il est encore au moins pour partie fondé sur une distinction fondamentale entre un statif et un 
processif (D. Cohen 1968, p. 1307 ; 1972, p. 58-59). Analyse également admise d'ailleurs depuis longtemps dans le champ des études berbères par Karl Prasse (1973, p. 29 et suiv.).

50 Non-orientation fondamentale du prédicat verbal, induisant une diathèse* centrée sur le morphème "transitivant-agentif" $s$-, ainsi qu'un système aspectuel fondé, pour un grand nombre de verbes, sur une opposition entre un "statif" et un "processif", sont autant de traits archaïsants de la syntaxe du verbe berbère qui devraient conduire à une réévaluation sensible de la position de cette langue dans la linguistique comparée et la reconstruction chamito-sémitiques.

$51 \rightarrow$ Voir aussi «Aspect» (A295, EB VII, 1989); "Dérivation» (D35, EB XV, 1995); « Diathèse » (D44, EB XV, 1995); « Verbe ».

\section{BIBLIOGRAPHIE}

ABDELMASSIH E. T., 1968 - Tamazight Verb Structure : a Generative Approach, Bloomington, Indiana University, The Hague, Mouton.

AIKHENVALD A., 1986 - "On the reconstruction of syntactic System in Berber-Libyc", Zeitschrift für Phonetik, Sprachwissenschaft und Kommunikation, 39 (5), p. 527-539.

AIKHENVALD A., 1985 - «Vers une caractérisation typologique des marques des constituants de phrase dans les langues libyco-berbères ", Lingvisticeskoe issledovanija. Tipologija. Etimologija. Komparativistika, Moscou, Nauka, p. 3-13.

AIKHENVALD A., 1985 - «Sur l'expression du patient avec pronoms personnels dans les langues libyco-berbères ", Tipy kommunikacii i coderzatel'nyi aspekt jazyka, Institut. Jazykoznanija AN SSSR, Moscou, Nauka, p. 57-58.

AIT-AHMED S., 1992 - « Un particularisme de Tamazight : les modalités "d" et "n" », Unité et diversité de Tamazight, I, [Colloque de Ghardaïa, 20-21 avril 1991], Tizi-Ouzou, Fnaca.

AKOUAOU A., 1976 - L'expression de la qualité en berbère : le verbe (parler de base : la tašelhit de Tiznit Thèse de Doctorat de $3^{\mathrm{e}}$ cycle, Paris.

APPLEGATE J. R., 1970 - “The Berber Languages”, Current Trends in linguistics, vol. 6, Paris/La Haye. ASPINION R., 1953 - Apprenons le berbère (Initiation aux dialectes chleuhs), Rabat, F. Moncho.

BASSET A., 1952 (1969) - La langue berbère, Londres.

BASSET A., 1957 - Articles de dialectologie berbère, Paris, Klincksieck.

BASSET A., Picard A., 1948 - Éléments de grammaire berbère (Kabylie - Irjen), Alger, La Typo-Litho/J. Carbonel.

BENTOLILA F., 1969 - « Les modalités d'orientation du procès en berbère. Aït seghrouchen », La Linguistique, $5 / 1$ et $5 / 2$.

BENTOLILA F., 1981 - Grammaire fonctionnelle d'un parler berbère, Paris, Selaf. 
BENVENISTE E., 1966/1974 - Problèmes de linguistique générale, I/II, Paris.

CADI K., 1987 - Système verbal rifain, forme et sens..., Paris, Peeters (Selaf).

CADI K., 1990 - Transitivité et diathèse en tarifit : analyse de quelques relations de dépendances lexicales et syntaxiques, thèse de doctorat d'Etat, Univ. Paris-III. Publié en 2006, Rabat, IRCAM.

CHAKER S., 1973 - Le système dérivationnel verbal berbère (dialecte kabyle), Paris, EPHE, Université René Descartes (thèse pour le doctorat de 3e cycle), 2 vol.

CHAKER S., 1983 - Un parler berbère d'Algérie (Kabylie) : syntaxe, Université de Provence.

CHAKER S., 1984 - Textes en linguistique berbère (introduction au domaine berbère), Paris, CNRS.

CHAKER S., 1995 - Linguistique berbère. Etudes de syntaxe et de diachronie, Paris/Louvain, Editions

Peeters ; notamment chap. 6 : «L'orientation du prédicat verbal ».

COHEN D., 1968 - « Les langues chamito-sémitiques », Le langage, Paris, NRF-Gallimard ("La

Pléïade").

COHEN D., 1984 - La phrase nominale et l'évolution du système verbal en sémitique. Etude de syntaxe historique, Leuven/Paris, Peeters.

COHEN D., 1989 - L'aspect verbal, Paris, PUF.

DALLET J.-M., Dictionnaire kabyle-français, Paris, 1982, p. 948.

DELHEURE J., 1987 - Grammaire de la Teggargrent berbère parlé à Ouargla, Paris, Pères Blancs, 143 p. (dactylographié).

DESTAING E., 1935 - « Note sur le verbe passif », C.R. du GLECS, 2, p. 31-32.

DIAKONOFF I. M.I., 1965/1988 - Semito-hamitic languages, Moscou. Réédition révisée : 1988 - Afrasian languages, Moscou, Nauka.

DUBOIS J. 1967 - Grammaire structurale du français. Le verbe, Paris, Larousse ("Langue et Langage").

DUBOIS J. et al., 1973 - Dictionnaire de linguistique, Paris.

FOUCAULD Ch. de, 1950-1952 - Dictionnaire touareg-français (dialecte de l'Ahaggar), Paris, Imprimerie Nationale, 4 tomes.

GALAND L., 1955 - « Etat et procès », Hesperis, 42, p. 245-251.

GALAND L., 1981 - « Le système verbal berbère » et « Problèmes de l'orientation du procès en berbère ", Actants, voix et aspects verbaux, Presses de l'université d'Angers.

GALAND L., 1987 - « Redistribution des rôles dans l'énoncé verbal en berbère », Actances [Paris/ Rivalc], 3.

GALAND L., 1998 - «Le berbère ", Les langues dans le monde ancien et moderne, $3^{\mathrm{e}}$ partie : Les langues chamito-sémitiques, Paris, CNRS.

GALAND L., 2002 - Etudes de linguistique berbère, Paris/Louvain, Peeters (Publications de la Société de Linguistique de Paris).

GALAND L., 2010 - Regards sur le berbère, Milan, Centro di Studi Camito-Semitici.

GUERSSEL M., 1986 - On Berber Verbs of Change, Cambrideg, MIT (Lexicon Project Working Papers, 9).

GUERSSEL M., 1987 - “Berber cauzadvization”, Current Approaches to African Linguistics IV, Dordrecht, Foris. 
GUERSSEL M. \& HALE K. (eds), 1985 - “A cross linguistic study of transitivity alternations”, Papers from the Regional Meetings [Chicago], 21/1.

GUERSSEL M. \& Hale Kenneth (eds), 1987 - Studies in Berber syntax, Cambridge, MIT.

LEGUIL A., 1992 - Structures prédicatives en berbère. Bilan et perspectives, Paris, L'Harmattan (1992.

MARTINET A., 1967 (4e édition) - Eléments de Linguistique Générale, Paris, A. Colin.

MARTINET A., 1968 (2 édition), La Linguistique synchronique, Paris, PUF.

MARTINET A., 1975 - Studies in Functional Syntax, Etudes de Syntaxe fonctionnelle, München, Wilhelm Fink Verlag.

MARTINET A., 1985 - Syntaxe générale, Paris, Armand Colin.

METTOUCHI A., 2005 - Contribution à l'étude de la prédication. De la sémantique énonciative à la typologie, Dossier (synthèse) pour l'Habilitation à Diriger des Recherches, Inalco, novembre 2005.

PENCHOEN Th. G., 1973a - Etude syntaxique d'un parler berbère (Aït Frah de l'Aurès), Napoli (= Studi Magrebini V).

PENCHOEN Th. G., 1973b - Tamazight of the Ayt Ndhir, Los Angeles.

PRASSE K.-G., 1972-2009 - Manuel de grammaire touarègue (tahaggart), Copenhague, Akademisk Forlag ; 1972 : I-III, Phonétique-Ecriture-Pronom, 1974 : IV-V, Nom ; 1973 : VI-VIII, Verbe ; Schwülper, Cargo Verlag, 2009 : VIII-IX, Syntaxe.

TAÏFI M., Dictionnaire tamazight-français (parlers du Maroc central), Paris, L’Harmattan, 1991.

TCHEKHOFF Cl., 1978 - Aux fondements de la syntaxe : l'ergatif Paris, PUF (“Le linguiste”).

TESNIÈRE L., 1965 ( $2^{\mathrm{e}}$ édition) - Eléments de syntaxe structurale, Paris, Klincksieck.

WILLMS A., 1972 - Grammatik der südlichen beraberdialekte (Süd-Marokko), Hamburg.

\section{NOTES}

1. Quelques rares parlers (en Kabylie, au Sahara...) peuvent ne pas connaître ces phénomènes d'attraction qui ont valu à ces morphèmes affixes du verbe la dénomination de "satellites du verbe".

2. Problème que Basset avait parfaitement perçu, sans lui apporter de réponse, quand il se demandait : «...pourquoi l'expression du passif par une forme dérivée quand, en base, [...] la forme simple par elle-même, a généralement les trois valeurs d'actif, passif et réfléchi ?» (1952, p. 13).

\section{INDEX}

Mots-clés : Linguistique 